Technical note

\title{
The effect of apoptotic cells on virus-specific immune responses detected using IFN-gamma ELISPOT
}

Kevin Lenders ${ }^{\mathrm{a},{ }^{*}}$, Benson Ogunjimi ${ }^{\mathrm{b},{ }^{*},}$, Philippe Beutels ${ }^{\mathrm{b}}$, Niel Hens ${ }^{\mathrm{b}, \mathrm{c}}$, Pierre Van Damme $^{b}$, Zwi N. Berneman ${ }^{a}$, Viggo F.I. Van Tendeloo ${ }^{a}$, Evelien L.J.M. Smits ${ }^{a}$

a Laboratory of Experimental Hematology, Vaccine \& Infectious Disease Institute, University of Antwerp, Antwerp University Hospital, Wilrijkstraat 10, B-2650 Edegem, Belgium

${ }^{\mathrm{b}}$ Centre for Health Economics Research and Modeling Infectious Diseases (CHERMID) \& Centre for the Evaluation of Vaccination (CEV), Vaccine \& Infectious Disease Institute, University of Antwerp, Universiteitsplein 1, B-2610 Wilrijk, Belgium

${ }^{\mathrm{C}}$ Interuniversity Institute for Biostatistics and Statistical Bioinformatics, Hasselt University, Agoralaan, B-3590 Diepenbeek, Belgium

*Both authors contributed equally.

Corresponding author:

Dr. Evelien L.J.M. Smits, Laboratory of Experimental Hematology, Vaccine \& Infectious Disease Institute, University of Antwerp (UA), Antwerp University Hospital (UZA), Wilrijkstraat 10, B-2650 Edegem, Belgium

Email: evelien.smits@uza.be; Tel: +32 38213584 ; Fax: +32 38214456

\section{Abbreviations :}

CMV, cytomegalovirus; ELISPOT, enzyme-linked immunosorbent assay; PBMC, peripheral blood mononuclear cells; SFC, spot-forming cells; VZV, varicella zoster virus. 


\section{ABSTRACT}

Interferon (IFN)- $\gamma$ ELISPOT can be used to monitor the magnitude of virus-specific cellular immune responses in vaccine trials. Often, IFN- $\gamma$ ELISPOT is performed with cryopreserved peripheral blood mononuclear cells (PBMC). However, it has not been well defined yet to what extent diminished cell viability of PBMC following cryopreservation affects IFN- $\gamma$ responses in ELISPOT.

Therefore, we assessed the influence of apoptotic cells on the number of spot-forming cells (SFC) in IFN- $\gamma$ ELISPOT using a gradient of UV-irradiated apoptotic PBMC and viral antigens derived from varicella zoster virus (VZV) and cytomegalovirus (CMV).

No SFC were observed when UV-irradiated apoptotic cells were stimulated with VZV or CMV antigens. Moreover, presence of apoptotic cells among viable $T$ cells hampered the detection of SFC following stimulation with VZV or CMV cell lysates, but not with CMVpp65 peptide pool. Statistical analysis showed that mainly late apoptotic cells, staining both Annexin $V$ and 7-amino-actinomycin $D$ (7-AAD), were associated with a decreased number of SFC.

In conclusion, it is recommended to use highly viable thawed PBMC for the detection of virus-specific cellular immune responses by IFN- $\gamma$ ELISPOT, since the detection of CMV- and VZV-specific $T$ cell responses stimulated by cell lysates was significantly impeded by the presence of apoptotic cells.

\section{Keywords:}

ELISPOT, apoptosis, IFN-gamma, vaccine trial, cellular immune response 


\section{INTRODUCTION}

The use of cryopreserved PBMC allows the determination of virus-specific immune responses in vaccine trials by the sensitive ELISPOT technique [1-4]. Generally, viability of PBMC after thawing is determined using DNA-intercalating agents, e.g. 7-aminoactinomycin $\mathrm{D}(7-\mathrm{A} A \mathrm{D})$ or propidium iodide $(\mathrm{PI})$, to identify dying or dead cells that have lost membrane integrity. However, cell viability following cryopreservation can be determined even more accurately when also a marker of apoptosis is included, e.g. Annexin V [5]. In this study, we wanted to assess the influence of apoptotic PBMC on the magnitude of virus-specific T cell responses against different forms of VZV- or CMVderived antigens using IFN- $\gamma$ ELISPOT. 


\section{MATERIALS AND METHODS}

\section{Isolation and cryopreservation of peripheral blood mononuclear cells}

Peripheral blood from healthy adult donors was collected by the Antwerp Blood Transfusion Center or by the Centre for the Evaluation of Vaccination (CEV, University of Antwerp). Within 24 hours after collection, PBMC were isolated by Ficoll-Paque Plus gradient separation (Amersham Biosciences, Sweden). All PBMC were frozen in $90 \%$ fetal bovine serum (FBS; Perbio Science, Belgium), supplemented with 10\% dimethyl sulphoxide (DMSO; Sigma-Aldrich, Germany) and stored at $-80^{\circ} \mathrm{C}$ or $-196^{\circ} \mathrm{C}$ (for longterm storage).

\section{Induction and determination of apoptosis}

Apoptosis was induced in thawed PBMC by UV irradiation. After an overnight resting phase, viability of the UV-irradiated and non-irradiated thawed PBMC was determined by flow cytometry after staining with Annexin V (FITC-conjugated; BD, Belgium) and 7-AAD (BD). Flow cytometry was performed on a FACScan (BD). Routinely, $1 \times 10^{4}$ events per target population were measured and analyzed using CellQuest software (BD) or FlowJo software version 7.2 (Tree Star, USA).

\section{Detection of IFN-Y-secreting cells by ELISPOT}

A gradient of UV-irradiated, apoptotic PBMC was added to a variable or fixed number of non-irradiated thawed PBMC of the same donor and IFN- $\gamma$ ELISPOT was performed on these PBMC in the presence of viral antigens. The antigens used for stimulation were cell lysates of MRC- 5 cells infected with VZV or CMV (Microbix Biosystems, Canada), as well as a CMVpp65 peptide pool (Miltenyi Biotec, Germany).

PBMC were stimulated in triplo for $18 \mathrm{~h}$ with virus-specific antigens in 96-well ELISPOT plates coated with IFN- $\gamma$ capture antibody (Millipore, Ireland). Positive controls consisted of PBMC stimulated with phytohemagglutinin (PHA; Sigma-Aldrich) and ionomycine (Sigma-Aldrich), negative controls of medium supplemented with BSA. ELISPOT assays were performed according to the manufacturer's instructions (Diaclone, France). Spotforming cells (SFC) were automatically counted by AID ELISPOT Reader System (AID 
Autoimmun Diagnostika, Germany) and analyzed using AID ELISPOT Software version 4.0. All SFC counted by the ELISPOT reader were manually audited to ensure that only true spots were included. Results are expressed as the number of SFC per $2.5 \times 10^{5}$ PBMC. The number of SFC was calculated by subtracting the mean background response from the mean response to the viral antigens. Responders were defined according to the guidelines of the ELISPOT proficiency panel IV CVC-2009, for the condition where no UV-irradiated PBMC were added: 1) mean of the replicates must be greater than or equal to 10 spots per well; and 2) mean of the replicates must be greater than three times the mean of the replicate from the background wells.

\section{Statistics}

Linear mixed models [6] were used to analyze the longitudinal variation in ELISPOT results when gradually adding apoptotic cells. Furthermore, we used Akaike's Information Criterion (AIC) [7] to select the best model out of a selection of candidate models. All results had $p<0.05$ unless stated otherwise. 


\section{RESULTS}

We performed IFN- $\gamma$ ELISPOT analysis to determine the number of SFC in a population of PBMC stimulated with viral antigens derived from CMV or VZV. Responders were defined according to the guidelines of the ELISPOT proficiency panel IV CVC-2009 (as described in Materials and Methods section).

First, we checked if we could detect SFC if only UV-irradiated PBMC were put into the ELISPOT well. As shown in Figure 1, no SFC cells were detected when UV-irradiated PBMC were stimulated with VZV lysate (Figure $1 \mathrm{~A}$ ), CMV lysate (Figure 1B) or CMVpp65 peptide pool (Figure 1C), although the same donors responded to these viral antigens when thawed, non-irradiated PBMC were used (Figure $1 \mathrm{~A}-\mathrm{C}$ ). When the fraction of UV-irradiated apoptotic cells was gradually increased within a total of $2.5 \mathrm{x}$ $10^{5}$ PBMC per well, the number of SFC in response to VZV- or CMV-antigen decreased, as expected because of the decrease in viable spot-forming PBMC (Figure $1 \mathrm{~A}-\mathrm{C}$ ). However, the VZV-specific response also decreased when increasing the amount of UVirradiated apoptotic cells added to a fixed number of viable PBMC per well in the presence of VZV lysate (Figure 2A). This decrease in the number of spots was less pronounced when PBMC were stimulated with CMV lysate (Figure $2 \mathrm{~B}$ ) and absent when using a CMVpp65 peptide pool as the source of antigen (Figure 2C).

The Linear Mixed Models (LMM) methodology was applied to further analyze the longitudinal variation in IFN- $\gamma$ T cell ELISPOT results when apoptotic cells were gradually added. In this statistical approach, the ELISPOT response was initially modelled as a linear function, E $=1+$ b.Grad, with $E$ the ELISPOT response, I the intercept and Grad the gradient of added apoptotic cells [from 0 (no addition of UVirradiated cells) to 100 (same amount of irradiated and non-irradiated cells per well)]. Table 1A shows the results for the different viral antigens. The best model for VZV lysate $(n=7)$ was $E=I+b . G r a d$ [with I including an effect of variation between individuals (random effect)]. This model shows that the ELISPOT response against VZV lysate will decrease with 0.27 spots on average when adding 2500 UV-irradiated PBMC. Also for CMV lysate $(n=11), E=I+b . G r a d$ was shown to be the best model, with a decrease of 
0.14 spots on average when adding 2500 UV-irradiated PBMC. However, for the CMVpp65 peptide pool $(n=21)$, the best model was $E=I$, meaning that no effect of apoptotic cells could be shown. In summary, this statistical analysis showed an effect of UV-irradiated cells on the amount of SFC in IFN- $\gamma$ ELISPOT only for VZV and CMV lysate, and not for the CMV peptide pool. Furthermore, this effect of the apoptotic cells was shown to be a general effect (no variation between individuals).

The model $E=1+b$.Grad was further decomposed taking into account different conditions of cell death: viable ( $\mathrm{V}^{;} 7-\mathrm{AAD}^{-}$and Annexin $\mathrm{V}^{-}$); early apoptotic (EA; 7-AAD and Annexin $\mathrm{V}^{+}$); late apoptotic $\left(\mathrm{LA} ; 7-\mathrm{AAD}^{+}\right.$and Annexin $\left.\mathrm{V}^{+}\right)$; and necrotic $\left(7-\mathrm{AAD}^{+}\right.$and Annexin $\mathrm{V}^{-}$). This model was explored for $\mathrm{VZV}$ and $\mathrm{CMV}$ lysate as $\mathrm{E}=\mathrm{a} \cdot \mathrm{V}+\mathrm{b} . \mathrm{EA}+$ c.LA, neglecting necrotic cells because of their relatively small numbers. The LMM analysis for VZV lysate yielded two similarly performing models, $E=a . V+b . E A+c . L A$; and $E=a . V+c . L A$. In the latter model, the ELISPOT response would decrease with 1 unit when adding 5263 late apoptotic cells. For CMV lysate also two similarly performing models emerged, $E=a . V+c . L A$ with $V$ and $L A$ containing random effects; and $E=a . V+c . L A$ with only $V$ containing random effects. The overall conclusion of the decomposition was the clear tendency towards signal-producing (individual-specific) effects of the viable cells, which were reduced mainly by the late apoptotic UV-irradiated cells, whereas the early apoptotic UV-irradiated cells were shown to be of minor importance (Table 1B). 


\section{DISCUSSION}

In this study, we showed that the presence of apoptotic cells in a population of thawed PBMC can affect virus-specific IFN- $\gamma$ T cell responses detected by ELISPOT. This effect was dependent both on the type of virus and on the type of antigen used for stimulation. Our data are in concordance with a recent study, which found the level of apoptosis to be the most indicative acceptance criterion (limit of $<18 \%$ ) to separate samples that could (effectively) respond to antigenic stimulation using T cell ELISPOT from samples that could not [8]. Our study clarifies these results by showing that UV-irradiated apoptotic cells not only do not respond to viral antigens in ELISPOT, but also that high presence of apoptotic cells (mainly cells that are positive for both Annexin $V$ and 7-AAD) among viable $T$ cells hampers the detection of SFC. In conclusion, we recommend using highly viable fractions of thawed PBMC in ELISPOT for an accurate detection of virusspecific cellular immune responses in vaccine trials. 


\section{ACKNOWLEDGEMENTS}

We would like to thank all blood donors, as well as the Antwerp Blood Transfusion Center and CEV (University of Antwerp) for their help in collecting blood.

This work was supported by the Methusalem program of the Flemish Government attributed to prof. dr. Herman Goossens (University of Antwerp, Vaccine \& Infectious Disease Insitute, Vaxinfectio), the Interuniversity Attraction Pole financement program (IAP \#P6/41) of the Belgian Government, the Antwerp University Concerted Research Action (BOF-GOA, grant no. 802) and by research grants of the University of Antwerp (grant no. 5/2145), the Fund for Scientific Research - Flanders (FWO, grant G.0370.08 and G.0082.08), the Institute for the Promotion of Innovation by Science and Technology in Flanders (IWT, grant no. 060081) and the Stichting tegen Kanker (\#SCIE2006-22). 


\section{REFERENCES}

1 Levin MJ, Smith JG, Kaufhold RM et al. Decline in varicella-zoster virus (VZV)-specific cellmediated immunity with increasing age and boosting with a high-dose VZV vaccine. J Infect Dis 2003;188:1336-1344.

2 Smith JG, Liu X, Kaufhold RM et al. Development and validation of a gamma interferon ELISPOT assay for quantitation of cellular immune responses to varicella-zoster virus. Clin Diagn Lab Immunol 2001;8:871-879.

3 Keating SM, Bejon $\mathrm{P}$, Berthoud $\mathrm{T}$ et al. Durable human memory $\mathrm{T}$ cells quantifiable by cultured enzyme-linked immunospot assays are induced by heterologous prime boost immunization and correlate with protection against malaria. J Immunol 2005;175:5675-5680.

4 Firbas $C$, Jilma B, Tauber $E$ et al. Immunogenicity and safety of a novel therapeutic hepatitis $\mathrm{C}$ virus (HCV) peptide vaccine: a randomized, placebo controlled trial for dose optimization in 128 healthy subjects. Vaccine 2006;24:4343-4353.

5 Fowke KR, Behnke J, Hanson C et al. Apoptosis: a method for evaluating the cryopreservation of whole blood and peripheral blood mononuclear cells. J Immunol Methods 2000;244:139-144.

6 Verbeke G, Molenberghs G. Linear mixed models for longitudinal data. 2001. Springer Verlag.

7 Akaike $\mathrm{H}$. Information theory as an extension of the maximum likelihood principle. Second International Symposium on Information Theory 1973, 267-281. Akademiai Kiado, Budapest.

8 Smith JG, Joseph HR, Green T et al. Establishing acceptance criteria for cell-mediatedimmunity assays using frozen peripheral blood mononuclear cells stored under optimal and suboptimal conditions. Clin Vaccine Immunol 2007;14:527-537. 


\section{TABLE 1}

Table 1. Linear mixed models analysis of longitudinal variation in IFN- $\gamma \mathrm{T}$ cell ELISPOT response when apoptotic cells were gradually added.

\section{A $\quad E=I+$ b.Grad}

\begin{tabular}{llll}
\hline Antigen & Model & $\begin{array}{l}\text { Random } \\
\text { Effect }\end{array}$ & AIC \\
\hline VZV lysate & $\mathrm{I}=36.5 \pm 4.7$ & $\mathrm{I}(\mathrm{p}=0.108)$ & 289 \\
CMV lysate & $\mathrm{b}=-0.272 \pm 0.046$ & $\mathrm{I}$ & 380 \\
& $\begin{array}{l}\mathrm{I}=82 \pm 21 \\
\mathrm{~b}=-0.139 \pm 0.036\end{array}$ & $\mathrm{I}$ & 791 \\
CMV peptide pool & $\begin{array}{l}\mathrm{I}=118 \pm 21 \\
\mathrm{~b}=0\end{array}$ & $\mathrm{I}$ &
\end{tabular}

B $\quad E=a . V+b . E A+c . L A$

\begin{tabular}{|c|c|c|c|}
\hline Antigen & Model & $\begin{array}{l}\text { Random } \\
\text { Effect }\end{array}$ & AIC \\
\hline \multirow[t]{2}{*}{ VZV lysate } & 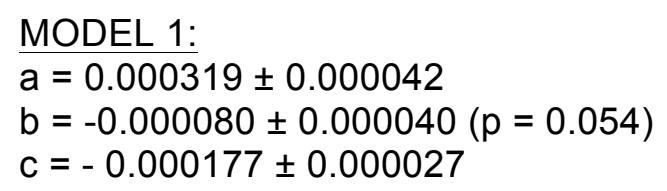 & $V(p=0.091)$ & 323 \\
\hline & $\begin{array}{l}\text { MODEL 2: } \\
\begin{array}{l}a=0.000279 \pm 0.000037 \\
b=0 \\
c=-0.000190 \pm 0.000027\end{array}\end{array}$ & $V(p=0.082)$ & 325 \\
\hline \multirow[t]{2}{*}{ CMV lysate } & $\begin{array}{l}\text { MODEL 1: } \\
\begin{array}{l}a=0.00060 \pm 0.00012 \\
b=0 \\
c=-0.000309 \pm 0.000062\end{array}\end{array}$ & $\begin{array}{l}\mathrm{V} \\
\mathrm{LA}(p=0.126)\end{array}$ & 505 \\
\hline & $\begin{array}{l}\text { MODEL 2: } \\
\begin{array}{l}a=0.00058 \pm 0.00012 \\
b=0 \\
c=-0.000238 \pm 0.000027\end{array}\end{array}$ & V & 510 \\
\hline
\end{tabular}


For each type of viral antigen, the best models (based on AIC) describing $E=I+b$.Grad (A) or $E=a . V+b . E A+c . L A(B)$ are shown with their respective values of fixed effects. $\mathrm{E}$, number of spot-forming cells in IFN- $\gamma$ ELISPOT; I, intercept; Grad, gradient; random effect, variables that contain both random and fixed effects; AIC, Akaike's Information Criterion; V, viable; EA, early apoptotic; LA, late apoptotic; VZV, varicella zoster virus; CMV, cytomegalovirus. All results have $p<0.05$ unless stated otherwise. 


\section{FIGURE LEGENDS}

Figure 1. Effect of UV-irradiation on IFN- $\gamma$ virus-specific $T$ cell ELISPOT response for PBMC stimulated with VZV cell lysate (A), CMV lysate (B) or CMVpp65 peptide pool (C). Each graph is a representative example showing the mean \pm SD of 1 donor for experiments done with 6 different responders to VZV and/or CMV antigens.

Figure 2. Effect of a gradient of UV-irradiated PBMC added to a fixed amount of viable PBMC on IFN- $\gamma$ virus-specific T cell ELISPOT responses. Cells were stimulated with VZV cell lysate (A), CMV lysate (B) or CMVpp65 peptide pool (C). Each graph is a representative example showing the mean \pm SD of 1 donor for experiments done with at least 6 different responders per type of antigen. 


\section{FIGURE 1}

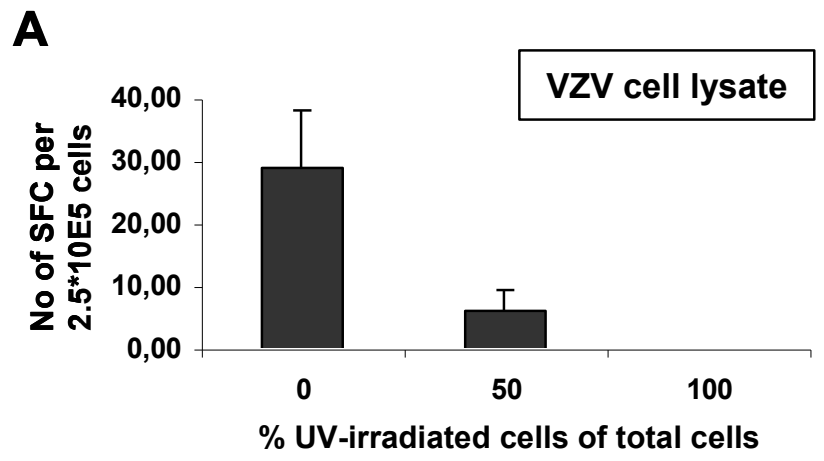

B
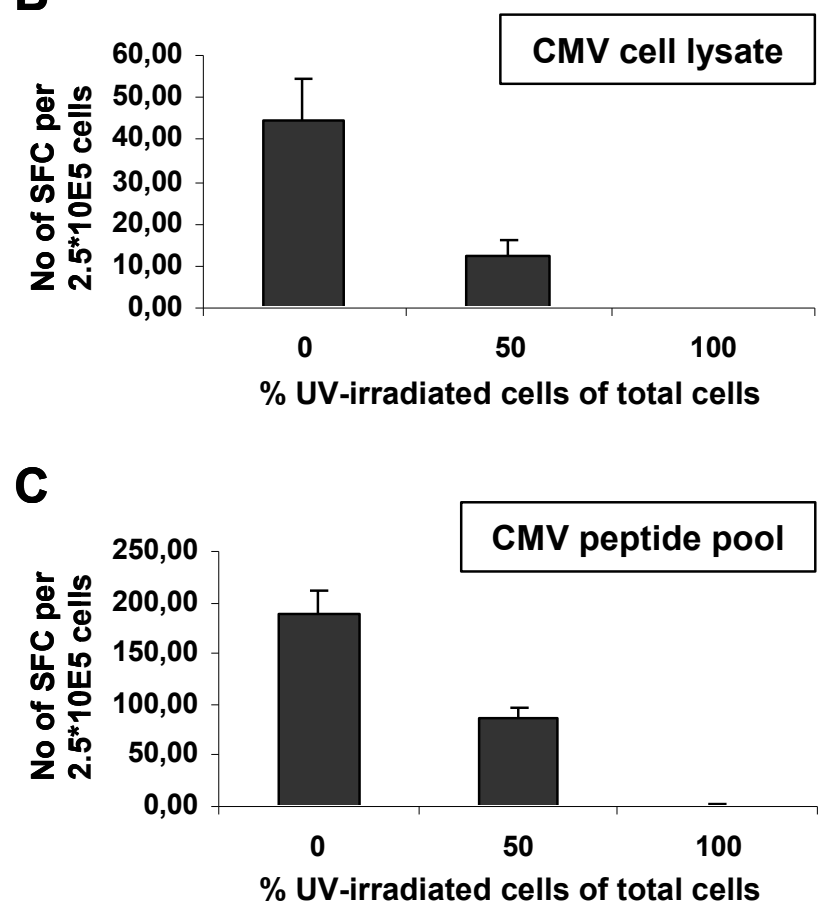


\section{FIGURE 2}
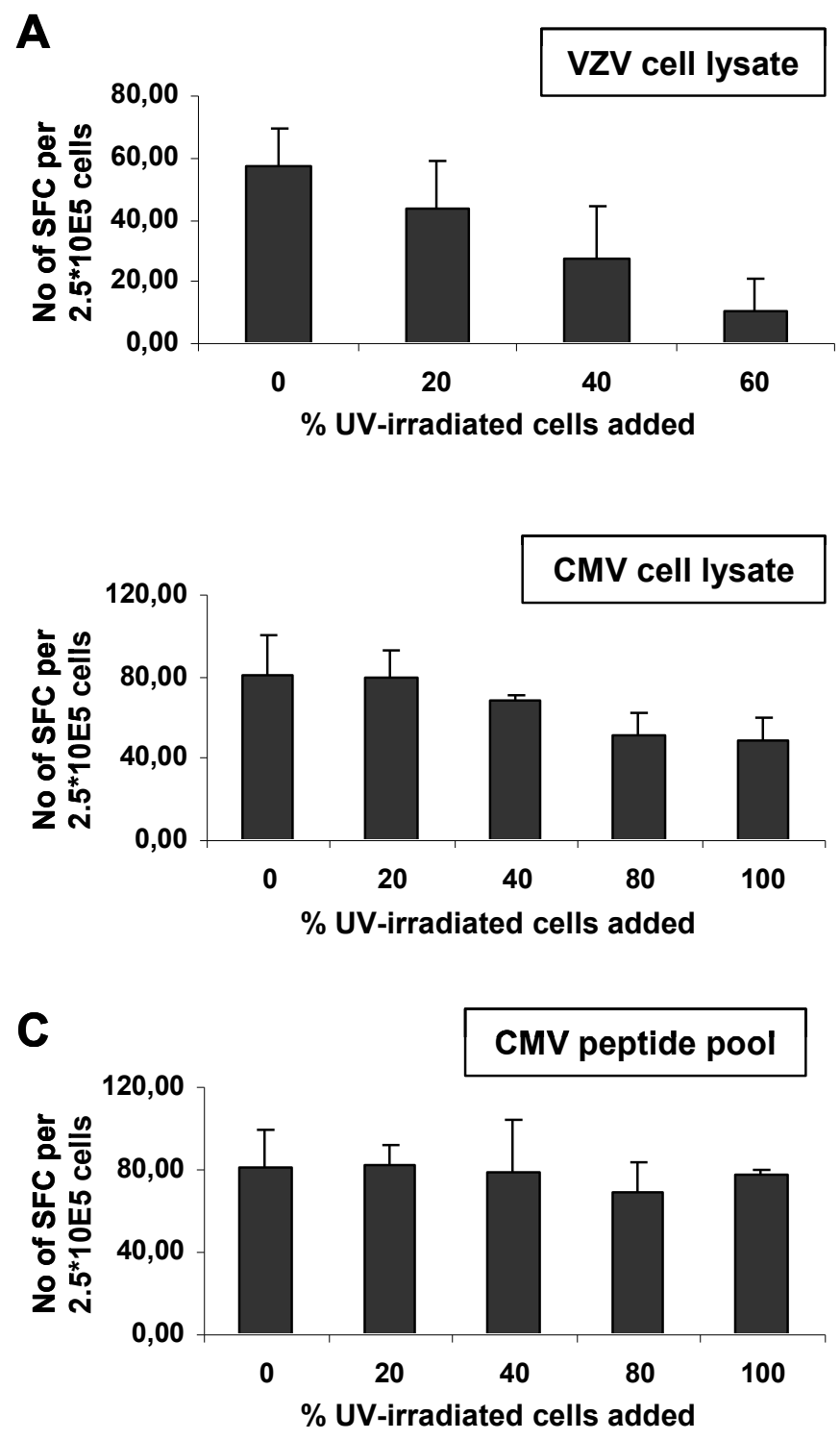